\title{
Initial effects of inflammation-related cytokines and signaling pathways on the pathogenesis of post-traumatic osteoarthritis ${ }^{\dagger}$
}

Review article

Peng-Fei Hana,b,\#, Zhi-Liang Zhang ${ }^{\mathrm{b}, \#}$, Tao-Yu Chen ${ }^{\mathrm{b}}$, Rui-Peng Zhao ${ }^{\mathrm{b}}$, Rong Zhang ${ }^{\mathrm{c}}$,

Xiao-Dong Lia, Peng-Cui Lib, Lei Wei ${ }^{b}$, Zhi Lv' ${ }^{b}$, Xiao-Chun Weib,*

a Second People's Hospital of Changzhi City, Changzhi, Shanxi 046010, China

${ }^{b}$ Second Affiliated Hospital of Shanxi Medical University, Taiyuan, Shanxi 030001, China

${ }^{c}$ Affiliated Peace Hospital of Changzhi Medical College, Changzhi, Shanxi 046010, China

Received: 2 January 2018; Accepted: 30 January 2018; Published: 20 June 2018

\begin{abstract}
The main pathological change in post-traumatic osteoarthritis (PTOA) is cartilage degeneration, which is closely related to inflammation and oxidative stress. Inflammation can cause degeneration of articular cartilage. Cartilage degeneration can also stimulate the progression of inflammation. It has been found that inflammatory cytokines can participate in the pathological process of cartilage degeneration through multiple signaling pathways, mainly mitogen-activated protein kinase, nuclear transcription factor kappa B, and Wnt- $\beta$-catenin signal transduction pathways. This review aimed at exploring the relationship between PTOA and inflammation-related cytokines by introducing the role of proinflammatory cytokines in chondrocyte destruction and extracellular matrix degradation.
\end{abstract}

Keywords: post-traumatic osteoarthritis • cartilage degeneration • proinflammatory cytokine • signaling pathway

(c) Shanxi Medical Periodical Press.

\section{Introduction}

Post-traumatic osteoarthritis (PTOA) is a common disease involving various tissues such as articular cartilage, subchondral bone, and synovium. It is a result of biodegradation and an imbalance in the synthesis of chondrocytes, extracellular matrix (ECM), and subchondral bone under the combined action of mechanical and biological factors. The typical pathological changes in PTOA are articular cartilage destruction and secondary bone hyperplasia, which are the consequences of the comprehensive effect of various factors. Studies have confirmed that nuclear transcription factor

† This project was supported by Shanxi Province Returned Overseas Students Research Funding Project (No. 2016-118).

\#Peng-Fei Han and Zhi-Liang Zhang contributed equally to this study.

*E-mail:sdeygksys@163.com kappa B (NF-kB), mitogen-activated protein kinase (MAPK), and Wnt- $\beta$-catenin signaling pathways and related proinflammatory cytokines [such as interleukins (ILs) and tumor necrosis factor (TNF- $\alpha$ )] as well as matrix metalloproteinases (MMPs) play crucial roles in the pathogenesis of PTOA. This review focuses on the signal transduction cascades and inflammatory cytokines relevant to the course of PTOA.

\section{PTOA-associated inflammation- related cytokines}

According to different cellular sources and biological characteristics, inflammation-related cytokines can be subdivided into proinflammatory cytokines and antiinflammatory cytokines. ${ }^{1,2}$ These two types of inflammation-related cytokines interact under pathological 
conditions, resulting in the imbalance of degeneration and synthesis during PTOA.

\subsection{Proinflammatory cytokines including IL-1, IL-6, IL-17, IL-18, and TNF- $\alpha$}

IL-1 is mainly synthesized by monocytes, although it can also be produced by fibroblasts, chondrocytes, macrophages, synoviocytes, and endothelial cells. Normal synovial fluid contains a trace amount of IL-1. This cytokine has two subtypes, IL-1 $\alpha$ and IL-1 $\beta$, and the latter is deeply involved in the pathogenesis of PTOA. ${ }^{3-5}$ Recent studies have indicated that IL-1 levels in synoviocytes and in the medium supernatant of chondrocytes from patients with PTOA and in mouse models are significantly increased in in vitro experiments, and IL-1 $\beta$ is mainly responsible. ${ }^{6,7}$ Attur et al. have shown that IL-1 $\beta$ is related to the severity of joint pain and radiological findings in patients with PTOA. ${ }^{8}$ Oligino et al. have induced typical PTOA-associated changes by transferring IL-1 $\beta$ into the synovial tissue of rabbit. ${ }^{9}$ Swellam et al. have demonstrated that expression of the gene encoding IL-1 correlates with the degree of cartilage degeneration in patients with PTOA. ${ }^{10}$ Some experimental studies have revealed that intra-articular injection of IL-1 $\beta$ receptor antagonists can significantly inhibit PTOA-associated synovial inflammation and hyperplasia, reduce the results of high-sensitivity C-reactive protein (CRP) assays, and enhance the synthesis of proteoglycans. ${ }^{11,12}$ Therefore, IL-1 can not only activate mononuclear macrophages and promote the expression of MMPs in cartilage and synovial tissues but also promote the growth of B lymphocytes and induce the expression of adhesion molecules on endothelial cells. Besides, it can inhibit the mRNA expression of pre-type II collagen in chondrocytes and consequently the synthesis of type II collagen and proteoglycan to ensure the functioning of bone resorption; IL-1 can also promote the synthesis and release of prostaglandin E2 (PGE2) and collagenase by synovial cells and chondrocytes. ${ }^{13}$ The formed PGE2 then enhances the inflammatory effect of IL-1, thus causing synovial inflammation and cartilage decomposition and absorption, resulting in the destruction of articular cartilage.

IL-6 is produced by lymphocytes, monocytes, osteophytes, and fat cells in the infrapatellar fat pad. It can stimulate synovial cells to proliferate and osteoclasts to activate and then causes the formation of synovial pannus and MMPs, eventually leading to the destruction of articular cartilage. Franco et al. ${ }^{14}$ have found that the levels of IL-6 in female blood can be used to predict the development of PTOA in the knee via the Kellgren-Lawrence Imaging score $(\mathrm{KL})$ scoring system. Hiraoka et al. have discovered that the levels of IL- 6 in osteoblasts of subchondral bone in patients with PTOA are significantly higher than those in patients without PTOA, and the level of IL-6 correlates with the formation of osteophytes and severity of this problem in patients with PTOA. ${ }^{15}$ Additionally, Livshits et al. have reported that the severity of unfavorable radiological findings in patients with PTOA is associated with the levels of IL-6 and CRP. ${ }^{16}$ Using an anti-IL-6R monoclonal antibody to inhibit IL-6R's downstream signal (Stat3) and serum IL-6 levels in a mouse Destabilization of the medial meniscus (DMM) model, Latourte et al. have found that synovial inflammation and cartilage damage are alleviated and osteophyte formation is reduced in a mouse model of PTOA. ${ }^{17}$

IL-17 is secreted by CD4 ${ }^{+}$memory T lymphocytes and monocytes. ${ }^{18}$ It can induce synovial fibroblasts, endothelial cells, and epithelial cells to secrete IL-6 and IL-8 and can enhance the proliferation of $\mathrm{T}$ lymphocytes. IL-17 can also stimulate the secretion of $\mathrm{IL}-1$ and TNF- $\alpha$ by macrophages in synovial fluid and increase the synthesis of PGE2, thereby promoting the expression of cyclooxygenase 2 (COX-2). Annunziato and Romagnani have concluded that the injection of IL-17 into the knee joint of mice can induce the onset of PTOA and increase synovial inflammation and destruction of articular cartilage. ${ }^{19}$ The abovementioned data indicate that IL-17 and IL-17R play a major part in the PTOA-associated imbalance of inflammatory factors and remodeling of connective tissue. Bush et al. have found that specific blocking of the action of endogenous IL-17 by means of soluble IL-17R-specific inhibitors can reduce the degree of an autoimmune reaction and joint damage and decrease the release of IL-6 and C-telopeptide (a type I collagen degradation marker) into synovium and bone tissue. ${ }^{20}$ Nitric oxide (NO) is a free radical that inhibits the adhesion of integrin $\beta 1$ to the ECM, reduces the synthesis of collagen and glycoproteins, and induces apoptosis in chondrocytes. Noone et al. have reported that IL-17 not only induces the production and release of $\mathrm{NO}$ but also synergizes with TNF- $\alpha$ and IL-10 in the induction of the MMP-13 release from osteoblasts and eventually causes ECM degradation. ${ }^{21}$

IL-18, a member of the IL-1 family, induces the production of interferon and granulocyte colony-stimulating factor by $\mathrm{T}$ lymphocytes, promotes the proliferation of $\mathrm{T}$ cells and Natural killer (NK) cells, and induces the production of MMPs, which are a group of endopeptidases that degrade the ECM and perform an important function in the destruction of cartilage tissue in patients with PTOA. At present, it is known that there are more than 10 MMPs involved in PTOA, and they can be classified into collagenases (MMP-1, -8, and -13), gelatinases (MMP-2 and -9), stromelysins (MMP-3, -7, -10, and -11), and other classes: a total of six categories. ${ }^{22}$ Some studies have confirmed that the amounts of MMP-1, $-2,-3,-7,-9$, and 
-13 increase in the joints of patients with PTOA. ${ }^{23,24}$ Overexpression of MMPs undermines the normal environment in which chondrocytes survive, leading to a decrease in the chondrocyte number. Damaged joints manifest thinning of cartilage, crevices, and biomechanical changes. Because MMP-1 is a fibroblast-type collagen hydrolase, it can directly degrade the most characteristic and abundant collagen in the ECM (type II collagen) and therefore crucially participates in the pathogenesis of PTOA.

TNF- $\alpha$ is mainly derived from monocyte macrophages. Its function and distribution in the body are similar to those of IL-1. TNF- $\alpha$ plays a key role in stromal degradation and cartilage destruction in PTOA. It can induce the production of other proinflammatory cytokines (such as IL-6), MMPs, and PGs and can downregulate proteoglycan and type II collagen. A high expression of TNF- $\alpha$ is detectable in the cartilage and synovium of patients with PTOA. Huang et al. have analyzed the expression of inflammatory factors and ECM degradation products in the plasma and cartilage of patients with PTOA and found that the expression of TNF- $\alpha$, MMP-3, and MMP-9 in these patients is significantly higher than that in the healthy people; the concentration of TNF- $\alpha$ in the synovial fluid is significantly higher too. ${ }^{25}$ Zwerina et al. have reported that progressive loss of proteoglycan and damage to cartilage tissue appear in the knees of TNF-overexpressing transgenic mice. ${ }^{26}$ Botha-Scheepers et al. have concluded that patients with higher levels of TNF- $\alpha$ in serum are at a sixfold higher risk of developing joint space narrowing (JSN) than those with lower levels of TNF- $\alpha .{ }^{27}$ Stannus et al. have shown that the level of serum TNF- $\alpha$ correlates with the JSN of the knee joint. ${ }^{28}$ Gerstenfeld et al. have treated mice manifesting damaged cartilage with TNF- $\alpha$, and after 24 hours, the researchers found that the proportion of chondrocyte apoptosis and death was as high as $50 \%$, whereas after elimination of TNF- $\alpha$ receptor, the phenomenon disappeared..$^{29}$ Brennan et al. have demonstrated that TNF- $\alpha$ receptor protein 55 is located intracellularly in the cartilage- and/or proteoglycan-deficient region of patients with PTOA ${ }^{30}$

In addition, IL-7, IL-8, IL-16, and other proinflammatory cytokines may be related to the initiation of PTOA.

\subsection{Anti-inflammatory cytokines including IL-4, IL-9, and IL-10}

IL-4 is an important protective cytokine in cartilage metabolism. Silvestri et al. have found that soluble Interleukin-4 receptor (IL-4R) concentration is significantly higher in the serum of patients with PTOA relative to healthy controls, and there is a correlation between the concentration of sIL-4R and severity of PTOA. ${ }^{31}$ IL-4 can inhibit IL-1 and thus perform a protective function for cartilage in vitro.

IL-9 can promote proliferation of chondrocytes and the synthesis of collagen and proteoglycan. Furthermore, IL-9 plays an important part in stimulating the body's self-repair in early PTOA.

IL-10 can inhibit the synthesis and secretion of IL-6 and other proinflammatory cytokines and has an immunomodulatory effect. ${ }^{32}$ Helmark et al. have discovered that PTOA patients' condition improves after exercise therapy, and this effect is associated with increased expression of IL-10. ${ }^{33}$

\section{PTOA-related signal transduction pathways}

In the joints of patients with PTOA, inflammatory cytokines deliver signals to downstream transcription factors through cell signaling pathways, thereby mediating the differentiation of chondrocytes into fibroblasts and reducing chondrocyte numbers in joints and alleviating fibrosis of the surrounding tissues. These signal transduction pathways include the NF- $\mathrm{KB}$ signaling cascade, the MAPK pathway, and the Wnt- $\beta$-catenin pathway. ${ }^{34-37}$

\subsection{The NF-KB signaling pathway}

The NF-KB family includes RelA (P65), RelB, c-Rel, NF- $\kappa B 1$ (P50), and NF-kB2. The most common of these is a heterodimer composed of $\mathrm{P} 65$ and $\mathrm{P} 50$, which are found in almost all types of cells in the human body. Both animal experiments and cell experiments indicate that the NF-KB signaling pathway is strongly activated in PTOA-affected joints. ${ }^{38,39}$ Activation of this pathway is mainly mediated by an upstream factor called the $I \kappa B$ kinase (IKK). When chondrocytes are stimulated by proinflammatory cytokines, oxidative stress, or other adverse stimuli, IKK is activated and the $I \kappa B$ protein (an antagonist of NF-KB) is phosphorylated and degraded, thereby promoting the expression of proinflammatory cytokines (IL-1, IL-6, IL-8, and TNF- $\alpha$ ) and MMPs. ${ }^{40}$ TNF- $\alpha$ and IL- 1 then activate the NF- $\kappa B$ pathway and upregulate the expression of MMP-1, MMP-3, and MMP-13 at mRNA and protein levels, thus accelerating degradation of the ECM and destruction of cartilage. In addition, studies have revealed that NF-кB can promote the production of $\mathrm{NO}$ and then induce apoptosis of chondrocytes. ${ }^{41}$

\subsection{MAPK signaling}

MAPKs are serine/threonine protein kinases that are widespread in eukaryotic cells. The MAPK signaling pathway is a three-stage kinase phosphorylation 
cascade, which is present in a wide range of mammalian cells and participates in cellular pathophysiology. ${ }^{42}$ This pathway can be triggered by extracellular signalregulated protein kinase, c-Jun $\mathrm{N}$-terminal kinase (JNK), stress-activated protein kinase, or P38. Among them, PTOA is mainly associated with p38 and JNK.

P38-activated MAPK signaling is crucial for the destruction of articular cartilage and can promote chondrocyte apoptosis, affect the synthesis of ECM of the cartilage, accelerate the processes of chondrocyte hypertrophy and calcification, and enhance the synthesis of MMPs. ${ }^{43}$ Various extracellular stimuli (such as extracellular environmental changes and inflammation-related cytokines) can cause phosphorylation of p38 MAPKs, which are activated and translocated into the nucleus and then stimulate the activation of transcription factor 2 (ATF-2) and muscle myocyte enhancer factor 2c (MEF2C). After activation, ATF-2 can bind NF- $\mathrm{KB}$ in the activation protein 1 site and drive the expression of proinflammatory cytokines (e.g., IL-1, TNF- $\alpha$ ) and MMPs. The phosphorylation of MEF2C can increase the expression of c-Jun, a transcription factor, which can bind to the docking site of activating protein 1 , thereby further enhancing the expression of inflammation-related genes. Moreover, upregulation of IL-1 and TNF- $\alpha$ can stimulate the phosphorylation of p38 MAPK, and therefore, a cascade effect (rapid enhancement) takes place to promote the production of COXs, PGE2, and MMPs, which inhibit the synthesis of ECM, thus aggravating the damage to chondrocytes. ${ }^{44}$ Moreover, Zhou et al. have shown that JNK can be activated when TNF- $\alpha$ continues to affect chondrocytes. In this process of chondrocyte apoptosis, the expression of apoptosis-inhibiting proteins (such as B-cell lymphoma-2 (Bcl-2) and myeloid cell leukemia-1 (Mcl-Bcl-2 and Mcl-1) appears to be significantly downregulated. ${ }^{45}$

\subsection{The Wnt- $\beta$-catenin signal transduction pathway}

This pathway involves a ligand (Wnt family of molecules), the Frizzled family, and low-density lipoprotein

\section{References}

1. Appleton CT. Osteoarthritis year in review 2017: Biology. Osteoarthritis Cartilage. 2018;26:296-303.

2. Watt FE. Osteoarthritis biomarkers: Year in review. Osteoarthritis Cartilage. 2018;26:312-318.

3. Abarca-Buis RF, Martínez-Jiménez A, Vera-Gómez $E$, et al. Mechanisms of epithelial thickening due to IL-1 signalling blockade and TNF- $\alpha$ administration
(LDL) receptor-related proteins 5 and 6 . Liu et al. have demonstrated that activation of Wnt- $\beta$-catenin signaling can promote differentiation of chondrocytes and reduce the synthesis of the ECM. ${ }^{46}$ At the same time, activation of the Wnt- $\beta$-catenin signaling pathway upregulates the mRNA expression of MMP-3 and MMP-13 and enhances the effect of IL-1 $\beta$, and these proinflammatory proteins further promote the apoptosis of chondrocytes ${ }^{47}$ Nevertheless, there are a few studies on the relation between the Wnt- $\beta$-catenin pathway and PTOA, and the specific mechanism underlying this link has yet to be studied.

\section{Conclusions}

In summary, inflammatory cytokines and their associated signal transduction pathways are closely linked to the pathogenesis of PTOA. Nonetheless, not all inflammatory cytokines are harmful, and we should correctly understand their pros and cons. It is believed that when the signal transduction mechanisms in PTOA are understood in more detail, researchers will come up with more ideas and ways for diagnosis and treatment of early PTOA (Figure 1).

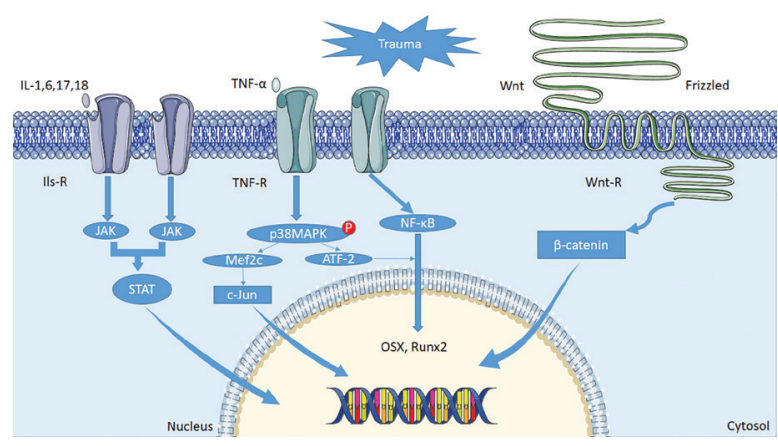

Figure 1. The role of various inflammatory factors and signal pathways in PTOA

\section{Conflicts of interest}

All contributing authors declare no conflicts of interest. differ during wound repair and regeneration. Differentiation. 2018;99:10-20.

4. Okla M, Zaher W, Alfayez M, Alfayez M, Chung S. Inhibitory effects of toll-like receptor 4, NLRP3 inflammasome, and interleukin-1 $\beta$ on white adipocyte browning. Inflammation. 2017;2:626-642. doi:10. 1007/s10753-017-0718-y. 
5. Shen HB, Huo ZJ, Bai YJ, et al. Protective effect of norcantharidin on collagen-induced arthritis rats. Chin J Integr Med. 2018;24:278-283. doi:10.1007/ s11655-017-2792-2.

6. Hwang HS, Park SJ, Lee MH, et al. MicroRNA-365 regulates IL-1 $\beta$-induced catabolic factor expression by targeting HIF-2 $\alpha$ in primary chondrocytes. Sci Rep. 2017;7:17889.

7. Zhou ZB, Du D, Chen A, Zhu L. Circular RNA expression profile of articular chondrocytes in an IL-1 $\beta$-induced mouse model of osteoarthritis. Gene. 2018;644:20-26.

8. Attur M, Statnikov A, Samuels J, et al. Plasma levels of interleukin-1 receptor antagonist (IL1Ra) predict radiographic progression of symptomatic knee osteoarthritis. Osteoarthritis Cartilage. 2015;23:1915-1924.

9. Oligino T, Ghivizzani S, Wolfe D, et al. Intra-articular delivery of a herpes simplex virus IL-1Ra gene vector reduces inflammation in a rabbit model of arthritis. Gene Ther. 1999;6:1713-1720.

10. Swellam M, Gabal KM, Youssef SS. Interleukin-1 receptor antagonist gene polymorphism and hepcidin in rheumatoid arthritis: Correlations with clinical and laboratory indices of disease activity. IUBMB Life. 2013;65:883-888

11. Martín-Sánchez F, Martínez-García JJ, MuñozGarcía M, et al. Lytic cell death induced by melittin bypasses pyroptosis but induces NLRP3 inflammasome activation and IL-1 $\beta$ release. Cell Death Dis. 2017;8:e2984.

12. Xing D, Gao H, Liu Z, Zhao Y, Gong M. Baicalin inhibits inflammatory responses to interleukin-1 $\beta$ stimulation in human chondrocytes. J Interferon Cytokine Res. 2017;37:398-405.

13. Takemiya T, Takeuchi C, Kawakami M. Microsomal prostaglandin e synthase-1 facilitates an intercellular interaction between CD4+ T cells through IL-1 $\beta$ autocrine function in experimental autoimmune encephalomyelitis. Int J Mol Sci. 2017;18:2758.

14. Franco L, Williams FM, Trofimov S, Surdulescu G, Spector T, Livshits G. Elevated plasma fractalkine levels are associated with higher levels of IL-6, Apo-B, LDL-C and insulin, but not with body composition in a large female twin sample. Metabolism. 2013;62:1081-1087.

15. Hiraoka N, Takahashi KA, Arai Y, et al. Intra-articular injection of hyaluronan restores the aberrant expression of matrix metalloproteinase-13 in osteoarthritic subchondral bone. J Orthop Res. 2011;29:354-360.

16. Livshits G, Zhai G, Hart DJ, et al. Interleukin-6 is a significant predictor of radiographic knee osteoarthritis: The Chingford Study. Arthritis Rheum. 2009;60:2037-2045.
17. Latourte A, Cherifi C, Maillet J, et al. Systemic inhibition of IL-6/Stat3 signalling protects against experimental osteoarthritis. J Ann Rheum Dis. 2017;76:748-755.

18. Li YS, Luo W, Zhu SA, Lei GH. T cells in osteoarthritis: Alterations and beyond. Front Immunol. 2017;8:356.

19. Annunziato F, Romagnani S. Mouse T helper 17 phenotype: Not so different than in man after all. Cytokine. 2011;56:112-115.

20. Bush KA, Farmer KM, Walker JS, Kirkham BW. Reduction of joint inflammation and bone erosion in rat adjuvant arthritis by treatment with interleukin-17 receptor IgG1 Fc fusion protein. Arthritis Rheum. 2002;46:802-805.

21. Noone C, Parkinson M, Dowling DJ, et al. Plasma cytokines, chemokines and cellular immune responses in pre-school Nigerian children infected with Plasmodium falciparum. Malar J. 2013;12:5.

22. Mobasheri A, Rayman MP, Gualillo O, Sellam J, van der Kraan P, Fearon U. The role of metabolism in the pathogenesis of osteoarthritis. Nat Rev Rheumatol. 2017;13:302-311.

23. Minkwitz S, Schmock A, Kurtoglu A, et al. Timedependent alterations of MMPs, TIMPs and tendon structure in human achilles tendons after acute rupture. Int J Mol Sci. 2017;18:pii:E2199.

24. Adams SB, Setton LA, Bell RD, et al. Inflammatory cytokines and matrix metalloproteinases in the synovial fluid after intra-articular ankle fracture. Foot Ankle Int. 2015;36:1264-1271.

25. Huang TL, Yang CH, Yanai G, Liao JY, Sumi S, Yang KC. Synergistic effect of I-ascorbic acid and hyaluronic acid on the expressions of matrix metalloproteinase-3 and -9 in human chondrocytes. J Biomed Mater Res B Appl Biomater. 2017 Sep 15. doi:10.1002/jbm.b.33988.

26. Zwerina J, Redlich K, Polzer K, et al. TNF-induced structural joint damage is mediated by IL-1. Proc Natl Acad Sci U S A. 2007;104:11742-11747.

27. Botha-Scheepers S, Watt I, Slagboom E, et al. Innate production of tumour necrosis factor alpha and interleukin 10 is associated with radiological progression of knee osteoarthritis. Ann Rheum Dis. 2008;67:1165-1169.

28. Stannus $\mathrm{O}$, Jones $\mathrm{G}$, Cicuttini F, et al. Circulating levels of IL- 6 and TNF- $\alpha$ are associated with knee radiographic osteoarthritis and knee cartilage loss in older adults. Osteoarthritis Cartilage. 2010;18:1441-1447.

29. Gerstenfeld LC, Cho TJ, Kon T, et al. Impaired intramembranous bone formation during bone repair in the absence of tumor necrosis factor-alpha signaling. Cells Tissues Organs. 2001;169:285-294. 
30. Brennan FM, Gibbons DL, Cope AP, Katsikis P, Maini RN, Feldmann M. TNF inhibitors are produced spontaneously by rheumatoid and osteoarthritic synovial joint cell cultures: Evidence of feedback control of TNF action. Scand J Immunol. 1995;42:158-165.

31. Silvestri T, Pulsatelli L, Dolzani P, Facchini A, Meliconi R. Elevated serum levels of soluble interleukin-4 receptor in osteoarthritis. Osteoarthritis Cartilage. 2006;14:717-719.

32. Campana L, Starkey Lewis PJ, Pellicoro A, et al. The STAT3-IL-10-IL-6 pathway is a novel regulator of macrophage efferocytosis and phenotypic conversion in sterile liver injury. J Immunol. 2018;200:1169-1187.

33. Helmark IC, Mikkelsen UR, Børglum J, et al. Exercise increases interleukin-10 levels both intraarticularly and peri-synovially in patients with knee osteoarthritis: A randomized controlled trial. Arthritis Res Ther. 2010;12:R126.

34. Ping Z, Wang ZR, Shi JW, et al. Inhibitory effects of melatonin on titanium particle-induced inflammatory bone resorption and osteoclastogenesis via suppression of NF-kB signaling. Acta Biomater. 2017;62:362-371.

35. Hou SM, Hou CH, Liu JF. CX3CL1 promotes MMP-3 production via the CX3CR1, c-Raf, MEK, ERK, and NF-KB signaling pathway in osteoarthritis synovial fibroblasts. Arthritis Res Ther. 2017;19:282.

36. Huang XJ, Pan QY, Mao ZK, et al. Sinapic acid inhibits the IL-1 $\beta$-induced inflammation via MAPK downregulation in rat chondrocytes. Inflammation. 2017 Dec 14. doi:10.1007/s10753-017-0712-4.

37. Yang XT, He HC, Gao Q, He CQ. Pulsed electromagnetic field improves subchondral bone microstructure in knee osteoarthritis rats through a Wnt/ß-catenin signaling-associated mechanism. Bioelectromagnetics. 2018;39:89-97. doi:18. 10.1002/bem.22106.

38. Pan TL, Shi XC, Chen $H$, et al. Geniposide suppresses interleukin-1 $\beta$-induced inflammation and apoptosis in rat chondrocytes via the PI3K/Akt/
NF-kB signaling pathway. J. Inflammation. 2017 Dec 6. doi:10.1007/s10753-017-0694-2.

39. Zheng W, Chen C, Zhang C, Cai L, Chen H. The protective effect of phloretin in osteoarthritis: An in vitro and in vivo study. Food Funct. 2018;9:263-278.

40. Hu H, Yang B, Li Y, Zhang S, Li Z. Blocking of the $\mathrm{P} 2 \times 7$ receptor inhibits the activation of the MMP13 and NF-KB pathways in the cartilage tissue of rats with osteoarthritis. Int $J$ Mol Med. 2016;38: 1922-1932.

41. Zhou Y, Ming J, Li Y, et al. Surfactant protein D attenuates nitric oxide-stimulated apoptosis in rat chondrocyte by suppressing p38 MAPK signaling. Biochem Biophys Res Commun. 2018;495:526-532.

42. Zeng $L$, Rong XF, Li RH, Wu XY. Icariin inhibits MMP1, MMP-3 and MMP-13 expression through MAPK pathways in IL-1 $\beta$-stimulated SW1353 chondrosarcoma cells. Mol Med Rep. 2017;15:2853-2858.

43. Chen WP, Jin GJ, Xiong Y, Hu PF, Bao JP, Wu LD. Rosmarinic acid down-regulates $\mathrm{NO}$ and PGE2 expression via MAPK pathway in rat chondrocytes. J Cell Mol Med. 2018;22:346-353.

44. Zhou Y, Liu SQ, Yu L, et al. Berberine prevents nitric oxide-induced rat chondrocyte apoptosis and cartilage degeneration in a rat osteoarthritis model via AMPK and p38 MAPK signaling. Apoptosis. 2015;20:1187-1199.

45. Liu S, Yang HP, Hu B, Zhang MY. Sirt1 regulates apoptosis and extracellular matrix degradation in resveratrol-treated osteoarthritis chondrocytes via the $W n t / \beta$-catenin signaling pathways. Exp Ther Med. 2017;14:5057-5062.

46. Weng LH, Wang CJ, Ko JY, Sun YC, Su YS, Wang FS. Inflammation induction of Dickkopf-1 mediates chondrocyte apoptosis in osteoarthritic joint. Osteoarthritis Cartilage. 2009;17:933-943.

47. Liao SY, Zhou K, Li DQ, Xie XM, Jun F, Wang J. Schisantherin A suppresses interleukin-1 $\beta$-induced inflammation in human chondrocytes via inhibition of NF-kB and MAPKs activation. Eur J Pharmacol. 2016;780:65-70.

How to cite this article: Han PF, Zhang ZL, Cheng TY, et al. Initial effects of inflammatory cytokines and signaling pathways on the pathogenesis of post-traumatic osteoarthritis. Front Nurs. 2018; 2: 91-96. https://doi. org/10.2478/fon-2018-0012 